\title{
Clinical Pharmacokinetics of Oral Sodium Selenite and Dosing Implications in the Treatment of Patients with Metastatic Cancer
}

\author{
Priya Jayachandran ${ }^{1}$ (D) Susan J. Knox ${ }^{2} \cdot$ Maria Garcia-Cremades $^{1} \cdot$ Radojka M. Savić $^{1}$
}

Accepted: 15 February 2021 / Published online: 17 April 2021

(c) The Author(s) 2021

\begin{abstract}
Background Selenite is a radiosensitizer and inhibitor of androgen receptor expression and function. In a phase I study (NCT02184533) in 15 subjects with metastatic cancer receiving daily oral sodium selenite with palliative radiation therapy, disease stabilization was observed, as evidenced by tumor regression, marked reduction in pain symptoms, and decreased prostate-specific antigen levels (only patients with castrate-resistant prostate cancer).

Objective The aim of this work was to characterize the pharmacokinetics of selenite to suggest dosing strategies and to propose a study design for further investigation.

Methods With selenium plasma concentrations obtained from five dosing cohorts $(5.5,11,16.5,33$, and $49.5 \mathrm{mg})$, a population pharmacokinetic model was constructed using NONMEM. The model described externally administered selenite (inorganic) with a baseline component for endogenous selenium levels. Using the pharmacokinetic model, simulations were performed to suggest dosing regimens that achieved in vitro target selenite levels, and optimal pharmacokinetic sampling times for a subsequent study were proposed using PopED.

Results A one-compartment model characterized selenite pharmacokinetics. Parameter estimates were absorption rate constant $\left(0.64 \mathrm{~h}^{-1}\right)$, apparent clearance $(1.58 \mathrm{~L} / \mathrm{h})$, apparent volume of distribution $(42.3 \mathrm{~L})$, and baseline selenium amount $(5270 \mu \mathrm{g})$. A logarithmic function characterized the inverse relationship between dose level and bioavailability. Four regimens to reach in vitro target selenite levels were proposed: $33 \mathrm{mg}$ daily, $16.5 \mathrm{mg}$ twice daily (BID), $11 \mathrm{mg}$ BID, and $5.5 \mathrm{mg}$ thrice daily (TID). Optimal sampling times were 1, 2, 6, and $24 \mathrm{~h}$.

Discussion The population model described the pharmacokinetic data well. Three regimens (33 mg daily, $11 \mathrm{mg}$ BID, 5.5 $\mathrm{mg}$ TID) achieved in vitro target selenite levels after one dose. The model and optimal sampling times may inform future studies evaluating the efficacy of selenite for metastatic cancer treatment.
\end{abstract}

\section{Introduction}

Androgens are male sex hormones that bind to androgen receptors (ARs) and mediate the growth and survival of prostate cells. Uncontrolled growth of these cells can lead to the development of prostate cancer. Lowering androgen levels and/or blocking the effect of androgens on AR signaling with androgen-deprivation therapy (ADT) reduces prostate cancer cell growth and delays disease progression [1]. ADT takes many forms (e.g., surgical removal of the

Priya Jayachandran

priya.jayachandran.pharmd.mse@gmail.com

1 Department of Bioengineering and Therapeutic Sciences, School of Pharmacy, University of California, San Francisco, 1700 4th Street, Room 501, San Francisco, CA 94158, USA

2 Department of Radiation Oncology, Stanford University School of Medicine, Stanford, CA, USA

\section{Key Points}

Patients with advanced or metastatic cancer often receive palliative radiation therapy, which frequently does not provide sufficient relief from disease symptoms.

Sodium selenite can slow the growth and spread of cancer cells by inducing cell death; it also makes tumor cells more sensitive to radiation therapy.

The proposed population model is the first of its kind to characterize the concentration-time course of selenite; the model may be used to suggest (1) dosing regimens and (2) optimal study designs for new clinical studies evaluating the efficacy of selenite as monotherapy or in combination therapy for treatment of advanced or metastatic cancer. 
testicles or ingestion of antiandrogen agents or female sex hormones) but, eventually, patients become resistant to ADT and develop castrate-resistant prostate cancer (CRPC) [2]. CRPC remains incurable despite recent advances in therapy that include new ADT agents and systemically targeted radiopharmaceutical treatments [3]. Radiation therapy (RT) is frequently used as palliation in CRPC; however, it often does not adequately alleviate symptoms such as pain caused by bone metastases. At this time, all treatments for CRPC are palliative at best, making the development of improved and more efficacious therapies for this disease a necessity [4].

Sodium selenite (hereafter referred to simply as selenite), is an inorganic form of selenium. Selenium is a trace element that is found naturally in foods, added to foods, and available as a supplement [5]. Selenite has antitumor effects in a variety of tumor types [6-10]. As selenite is metabolized, it depletes the antioxidant glutathione and generates superoxide radicals, which are toxic reactive oxygen species [6,7]. Reactive oxygen species can induce apoptosis and sensitize prostate tumor cells to the effects of radiation without sensitizing the normal gastrointestinal epithelium to radiation $[6,7]$. The preferential effect of selenite on prostate cancer cells (as compared with normal prostate cells) is due in part to alteration in the level of expression of the Bcl-2 family members, which are important regulators of apoptotic potential, as well as to baseline differences between cancerous and normal prostate cells in the level of the antioxidant enzyme manganese superoxide dismutase [6,7]. Furthermore, evidence suggests that selenite is a potentially novel inhibitor of AR expression and function and acts via a glutathionedependent redox mechanism to inhibit the transcription factor Sp1 (upstream of prostate-specific antigen and AR) [8, 9]. Taken together, these findings provide compelling evidence that selenite may be useful as a therapeutic agent for treating hormone-sensitive and refractory prostate cancer and other tumor types, either alone or in combination with standard cytotoxic and hormone therapies.

The clinical pharmacokinetics of intravenously administered selenite has been characterized using noncompartmental analysis. An elimination half-life of approximately $18 \mathrm{~h}$ was reported in patients with various primary tumor types [11]. These individuals experienced nausea and vomiting as the primary systemic toxicity at dose levels of $\geq 4.5 \mathrm{mg} / \mathrm{m}^{2}$ [11]. To characterize the safety and pharmacokinetics of orally administered selenite, a phase I study was conducted in patients with metastatic cancer, many of whom had CRPC [12]. Selenite was administered daily in combination with palliative RT for the duration of the RT course. Using a standard, nonrandomized, open-label design, patients with metastatic cancer were treated with escalating doses of selenite ranging from 5.5 to $49.5 \mathrm{mg}$, taken as a single oral dose without food $2 \mathrm{~h}$ prior to RT.
The (1) oral dosing regimens (dose, frequency) that may achieve target in vitro selenite levels and (2) optimal pharmacokinetic sampling times of oral selenite as an add-on therapy for patients with CRPC for whom treatment with other antiandrogens have failed is unknown. To explore these questions, we developed a population pharmacokinetic model using nonlinear mixed-effects modeling (NLMEM) with the pharmacokinetic data collected from plasma blood sampling for the various dosing cohorts. Selenite may be useful for treating not only prostate cancer but also other solid tumors and non-Hodgkin's lymphoma [11, 13-16]. Therefore, this quantitative approach and the primary findings from this study may be used to design and analyze similar phase I studies evaluating oral selenite as monotherapy or in combination therapy for patients with various types of metastatic cancer.

\section{Methods}

\subsection{Study Design, Population, and Pharmacokinetic Sampling}

The full study design and the primary efficacy and safety results are described elsewhere [12]. In brief, the study was a phase I, nonrandomized, open-label, single-stage trial to evaluate the efficacy and safety of oral selenite, in combination with palliative RT, in patients with metastatic cancer (NCT02184533). In total, 15 subjects (13 males, 2 females) aged 37-92 years received oral sodium selenite tablets administered without food ( $2 \mathrm{~h}$ before or after drug administration). Drug administration occurred approximately $2 \mathrm{~h}$ prior to scheduled daily RT sessions. Standard palliative regimens of RT were utilized, but the dose, fractionation, and duration varied according to a number of clinical factors. Therefore, not all patients received the same number of selenite doses, with some receiving up to ten doses. Selenium plasma concentrations were collected from each subject predose, at $15 \mathrm{~min}$, and at 1, 2, 4, and $24 \mathrm{~h}$ post-dose on day 1 (intensive sampling). All other sample collections on day 2 and thereafter were optional (sparse sampling). Of the dose escalation schema investigated, data for five dosing cohorts were available: $5.5,11,16.5,33$, and $49.5 \mathrm{mg}$ selenite (no control group was used). For the modeling analysis, the plasma concentrations collected in the intensive sampling phase for each individual in each dosing cohort were used.

\subsection{Population Pharmacokinetic Model Development and Evaluation}

A population approach using NONMEM software (version 7.4; ICON PLC, Dublin, Ireland) was used to model the selenite pharmacokinetics. A one-compartment and 
two-compartment model with oral absorption were tested. The two-compartment model did not significantly improve the model fit; therefore, a one-compartment structure was retained for the final model. A system of ordinary differential equations was written, and data were fit using the first-order conditional estimation method with interaction.

The time of selenite dose administration was approximately $2 \mathrm{~h}$ before RT and coincided with the first pre-dose/ baseline selenium measurement. A parameter was introduced into the model to estimate the baseline endogenous selenium amount (BASE) prior to dosing. The bioavailability $(F)$ was estimated for each dose level relative to $5.5 \mathrm{mg}$ $\left(F_{5.5}=\sim 1\right)$, which revealed a decrease in bioavailability with increasing dose level. To describe this dose-dependent bioavailability relationship, an empirical mathematical expression was fit to the bioavailability estimates per dose level; power, logarithmic, exponential, and linear functions were explored. The logarithmic relationship yielded the best fit and was subsequently used to replace the individual estimates of bioavailability per dose level in the model. A separate assessment using Michaelis-Menten saturable absorption kinetics was also explored. In addition to bioavailability and BASE, pharmacokinetic parameter estimates with uncertainty expressed as a relative standard error (RSE) were obtained for the absorption rate constant $\left(k_{\mathrm{a}}\right)$, apparent clearance $(\mathrm{CL} / \mathrm{F})$, and apparent volume of distribution $(V / F)$ of selenite. Interindividual variability $(\mathrm{IIV}, \eta)$ was modeled exponentially, added to each parameter $\left(k_{\mathrm{a}}, \mathrm{CL} / F\right.$, $V / F$, BASE), and assessed for model stability and convergence. Additive, proportional, and combined error models were evaluated to characterize the residual variability. IIV on the BASE parameter and an additive error model provided the best fit.

The best fit was determined based on likelihood ratio tests, diagnostic plots, and visual predictive checks (VPCs). The difference between two log likelihood ratios (-2LL), referred to as the objective function value (OFV), followed a chi-squared distribution; if the OFV difference between two models differing by a single parameter was $>3.84$, the parameter was deemed significant $(p<0.05)$. Diagnostic plots (goodness of fit and individual fits) and VPCs using 1000 simulated datasets was performed to both determine the appropriateness of the model and to suggest improvements. Perl-speaks-NONMEM (version 4.8.1), Pirana (version 2.9.7), $\mathrm{R}$ (version 3.5.1), and Xpose (version 0.4.4) were used for data management, model development, and data visualization [17].

\subsection{Proposed Dosing Regimens}

Using the final population pharmacokinetic model and its parameter estimates, typical pharmacokinetic simulations were performed for the dose levels studied in the trial
(5.5, 11, 16.5, 33, and $49.5 \mathrm{mg}$ selenite) to propose dosing regimens (dose, frequency) that achieved a target selenite concentration range of $5-10 \mu \mathrm{M}(395-790 \mathrm{ng} / \mathrm{mL}$ of selenium). This target was derived from in vitro studies because prior clinical efficacy data in patients are sparse [6-8]. The PKPDsim package (version 1.0.1) in $\mathrm{R}$ was used to perform simulations.

\subsection{Optimal Sampling Design}

To informatively design subsequent pharmacokinetic studies evaluating the efficacy of selenite for treatment of metastatic cancers, the optimal design software PopED (Population Experimental Design, version 0.4.0, R package) was used to determine optimal pharmacokinetic sampling times $[18,19]$. PopED uses a Fisher information matrix (FIM) for design optimization. The FIM defines the best design for parameter estimation using maximum likelihood estimation methods [20]. The central principle in FIM design optimization is D-optimality, where the determinant summarizing the FIM is maximized such that the precision of the parameter estimates is minimized for individual and population models [20].

A one-compartment model written with ordinary differential equations was used from the predefined structural model library. Initial conditions were set to zero for the absorption compartment and the BASE parameter for the central compartment. The logarithmic dose-dependent bioavailability expression was not used; instead, bioavailability at each dose level was expressed as $\mathrm{F} \times$ DOSE. From the final population pharmacokinetic model, the fixed-effect parameter estimates for $k_{\mathrm{a}}, \mathrm{CL} / F, V / F$, and BASE, and the IIV estimate on BASE were used. The residual variability was described by a predefined additive error model; the estimate reported from the final population pharmacokinetic model as a standard deviation was converted to a variance and used as the estimate for sigma. For the initial design, three groups of 25 individuals each with four sampling times per individual were defined; all groups were fixed to have the same sample times. The design space was defined with a range of potential sample times: $0-12 \mathrm{~h}$ for the first three samples and $12-24 \mathrm{~h}$ for the last sample. The initial design was simulated and evaluated. Subsequently, the sample times of the initial design were optimized within the design space for three dosing regimens: (1) $33 \mathrm{mg}$ daily, (2) $16.5 \mathrm{mg}$ twice daily (BID), and (3) $11 \mathrm{mg}$ BID. All the regimens were optimized simultaneously to provide a single set of sampling times that can be easily implemented in the clinical study setting. These regimens were selected with consideration for adherence (fewer doses per day) and toxicity (lower doses). Finally, the RSEs were compared for the parameter estimates obtained from NONMEM for the original design and PopED for the optimized design. 


\section{Results}

\subsection{Pharmacokinetic Data}

A total of 15 subjects provided pharmacokinetic data across five dosing cohorts; Table 1 in the electronic supplementary material (ESM) summarizes the patient characteristics per dosing cohort. Figure 1 illustrates the 24-h concentration-time profiles of the raw data for the study population, with a vertical line at the time of dose administration to delineate pre-dose from post-dose concentrations. The baseline selenium concentration range was $93-196 \mathrm{ng} / \mathrm{mL}$.

Selenite doses were administered to subjects before a scheduled RT session; since subjects 3 and 12 underwent a single RT session, they only received a single selenite dose and were not required to return to clinic for the 24-h sample collection. Subjects 15, 17, and 19 received more than one selenite dose, but they missed the 24-h sample collection after the first dose for logistical reasons.

\subsection{Population Pharmacokinetic Model and Evaluation}

Figure 2 provides a schematic of the population pharmacokinetic model. The schematic distinguishes the in vivo contributions of dietary selenium and the ex vivo contributions of exogenous selenite to the recorded plasma concentrations from the clinical study. The one-compartment model described the data well, and no significant model misspecifications were observed from the basic goodness-of-fit plots or the VPC (Fig. 3). The BASE was estimated $(5270 \mu \mathrm{g})$ and found to be in agreement with the raw data. Table 1 shows the other population pharmacokinetic parameter estimates $\left(k_{\mathrm{a}}, \mathrm{CL} / F, V / F\right)$ with residual standard errors from the final model. The dose-bioavailability relationship that provided the best

Table 1 Pharmacokinetic model parameter estimates

\begin{tabular}{lcl}
\hline Parameter & $\begin{array}{l}\text { Population estimate } \\
\text { (RSE, \%) }\end{array}$ & $\begin{array}{l}\text { Interindi- } \\
\text { vidual vari- } \\
\text { ability } \\
\% \mathrm{CV} \text { (RSE, } \\
\%)\end{array}$ \\
\hline$k_{\mathrm{a}}, \mathrm{h}^{-1}$ & $0.642(16)$ & - \\
$\mathrm{CL} / F_{5.5}, \mathrm{~L} / \mathrm{h}$ & $1.59(6)$ & - \\
$V / F_{5.5}, \mathrm{~L}$ & $42.5(10)$ & - \\
$\mathrm{BASE}, \mu \mathrm{g}$ & $5270(13)$ & $17(46)$ \\
Additive error, $\mu \mathrm{g} / \mathrm{L}$ & $55.9(10)$ & - \\
\hline
\end{tabular}

Bioavailability-dose function: $F=-0.229 \times \ln ($ DOSE $)+3.02$

$B A S E$ baseline selenium amount, $C L$ clearance, $C V$ coefficient of variation, $F$ bioavailability, $h$ hour, $k_{a}$ absorption rate constant, $R S E$ relative standard error, $V$ volume of central compartment fit for the bioavailability estimates per dose level was the logarithmic expression $F=-0.229 \times \ln ($ DOSE $)+3.02$. Using this relationship, the bioavailability at each dose level was 1.0, 0.9, 0.8, 0.6, and 0.5 for $5.5,11,16.5,33$, and $49.5 \mathrm{mg}$, respectively. These values are in agreement with the distinct bioavailability estimates for each dose level. The OFV decreased significantly with the inclusion of the BASE parameter, and the dose-dependent bioavailability relationship was also statistically significant.

\subsection{Proposed Dosing Regimens}

The most descriptive pharmacokinetic data available from the study were collected in the intensive sampling phase, which only provided concentrations following a single dose administration. Whether subjects in each dosing cohort were able to achieve a target in vitro selenite range of $5-10 \mu \mathrm{M}$ with the daily dosing was unknown. Therefore, simulations were performed using the population pharmacokinetic model to explore dosing regimens that do achieve the target in vitro selenite concentrations with the dose levels studied (Fig. 1 in the ESM). When selecting regimens to propose, consideration was given to the systemic toxicity (gastrointestinal) observed at $33 \mathrm{mg}$ (grade 1 and 2) and $49.5 \mathrm{mg}$ (grade 2) dose levels [12]. Therefore, the $49.5 \mathrm{mg}$ dose level and frequencies greater than daily for the $33 \mathrm{mg}$ dose level were not considered (Fig. 4).

Figure 1 in the ESM compares simulations following (1) single, (2) daily, (3) BID, and (4) three times daily (TID) administration at each dose level. Only higher dose levels (33 mg, $49.5 \mathrm{mg}$ ) achieved the target concentration range after a single dose. When given daily, the peak concentrations at the $49.5 \mathrm{mg}$ dose level exceeded the upper limit of the target concentration range $(790 \mathrm{ng} /$ $\mathrm{mL}$ ), whereas the trough concentrations at the $33 \mathrm{mg}$ dose level fell below the lower limit of the target concentration range $(395 \mathrm{ng} / \mathrm{mL}$ ). With BID administration, the middle dose levels $(16.5 \mathrm{mg}, 11 \mathrm{mg})$ fell well within the desired range, and TID dosing was necessary for the lowest dose level $(5.5 \mathrm{mg})$ to reach $395 \mathrm{ng} / \mathrm{mL}$. With consideration for toxicity, four dosing regimens are proposed: (1) $33 \mathrm{mg}$ daily, (2) $16.5 \mathrm{mg}$ BID, (3) $11 \mathrm{mg}$ BID, and (4) $5.5 \mathrm{mg}$ TID. An individual started on $33 \mathrm{mg}$ daily who experiences gastrointestinal side effects may safely deescalate to 16.5 or $11 \mathrm{mg}$ BID. Figure 4 illustrates these regimens for a time course of 1 week; Table 2 compares the trough and peak concentrations for each proposed regimen. The range of the trough and peak concentrations was widest for the $33 \mathrm{mg}$ daily regimen, and the highest peak and trough concentrations were achieved by the $16.5 \mathrm{mg}$ BID regimen. 


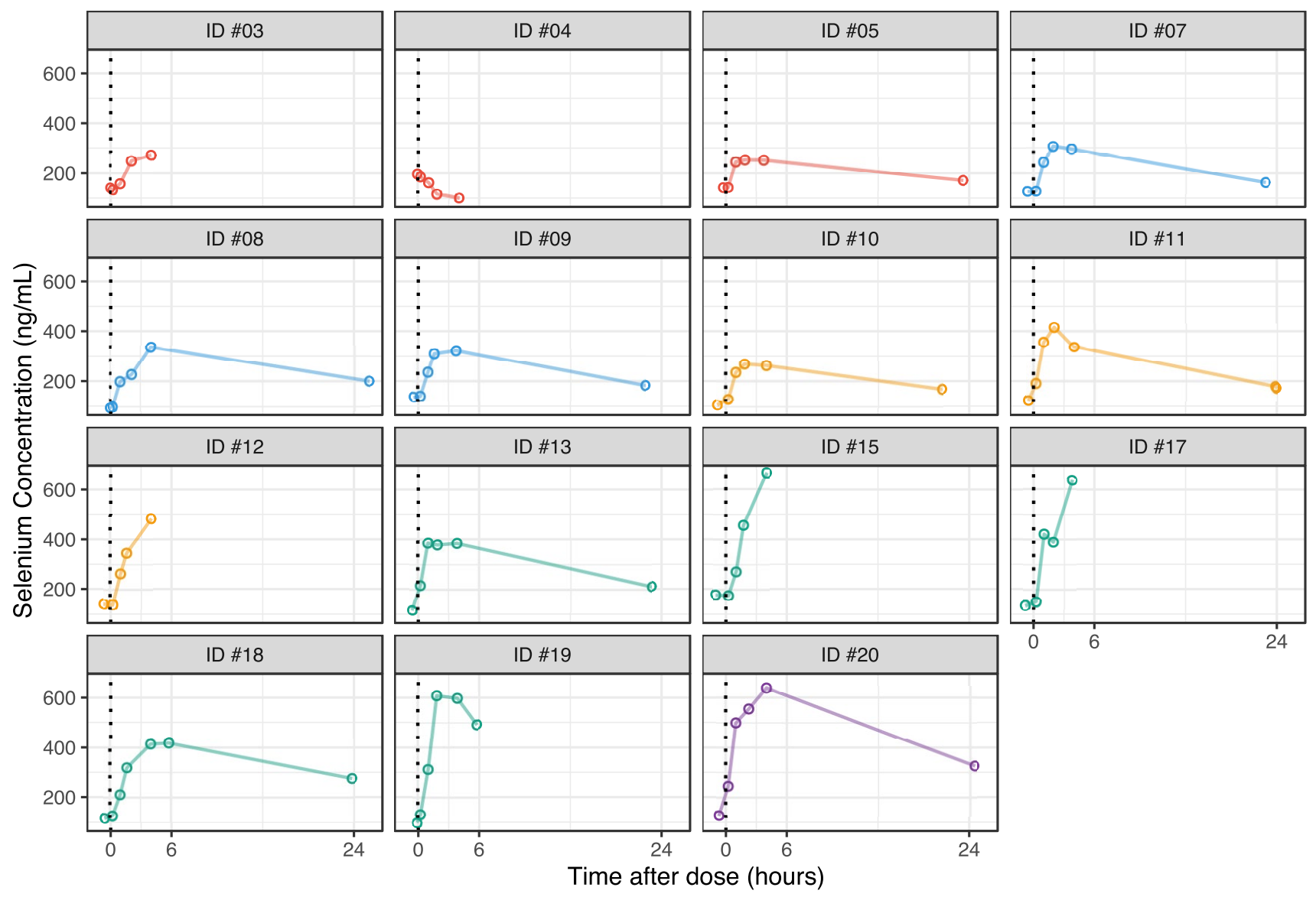

Dosing Cohort $-5.5 \mathrm{mg}=11 \mathrm{mg}=-16.5 \mathrm{mg}=-33 \mathrm{mg}$ - $-0-49.5 \mathrm{mg}$

Fig. 1 Pharmacokinetic profiles of raw data. Observed selenium concentration-time profiles for all 15 study subjects colored by dose level. Vertical dotted line denotes dose administration time

\subsection{Optimal Sampling Design}

The population pharmacokinetic model can be further utilized to propose the optimal pharmacokinetic sampling time points for a new pharmacokinetic study that evaluates the tolerability and efficacy of oral selenite in combination with ADT. Figure 5a illustrates the optimal experimental design for three dosing regimens: (1) $33 \mathrm{mg}$ daily, (2) $16.5 \mathrm{mg}$ BID, and (3) $11 \mathrm{mg}$ BID. The optimal pharmacokinetic sampling times for all three regimens are at 1, 2, 6, and $24 \mathrm{~h}$. Figure $5 \mathrm{~b}$ compares the precision, described using RSEs, of the pharmacokinetic parameter estimates for the original design and the optimized design. The RSEs are lower for the optimized design for all of the pharmacokinetic parameters except BASE; therefore, with the optimal sampling times, the precision of the parameter estimates can be improved. The highest RSE is for the BASE parameter; since the clinical pharmacokinetic study was not originally designed to estimate the baseline selenium level, a lower precision is not unexpected for this parameter.

\section{Discussion}

We constructed a first-of-its-kind population pharmacokinetic model using NLMEM to characterize the pharmacokinetics of oral selenite in plasma across a range of clinically studied doses. Using our model, we proposed dosing regimens that achieved target in vitro selenite levels and an optimal experimental study design for a subsequent pharmacokinetic study to evaluate the safety and efficacy of selenite as an add-on therapy for patients with CRPC for whom other treatments have failed.

Based on the pharmacokinetic parameter estimates obtained from the model, the calculated half-life is approximately $19 \mathrm{~h}$, which is in agreement with reports in the literature [11]. In the SECAR study, which evaluated intravenous selenite as monotherapy in patients with advanced cancer, Brodin et al. [11] reported a half-life of approximately $18 \mathrm{~h}$. The analysis utilized a noncompartmental approach 


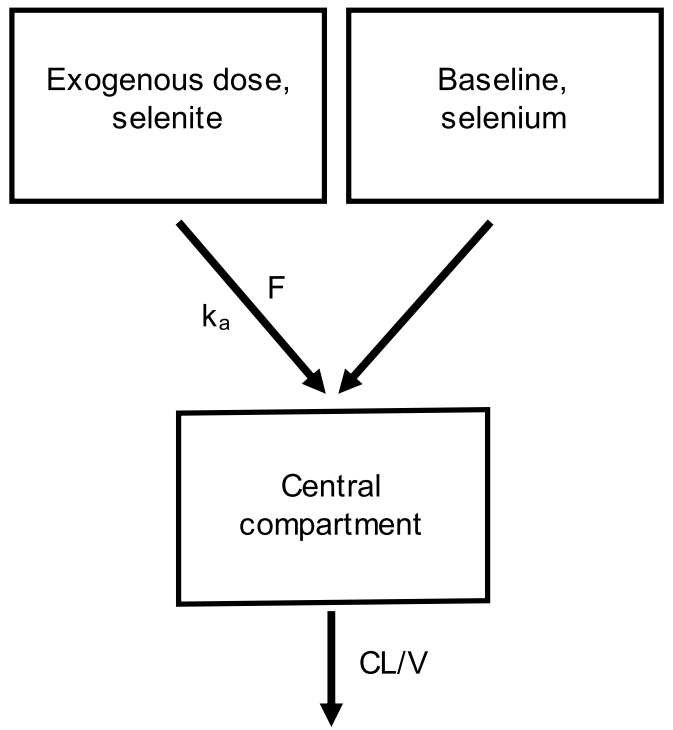

Fig. 2 Schematic of pharmacokinetic model. One-compartment pharmacokinetic model with absorption for oral administration of selenite. Following absorption $\left(k_{\mathrm{a}}\right)$, the exogenous dose is distributed into the systemic circulation (central compartment) from where it is eliminated $(\mathrm{CL} / \mathrm{V})$. Baseline comprises endogenous selenium levels

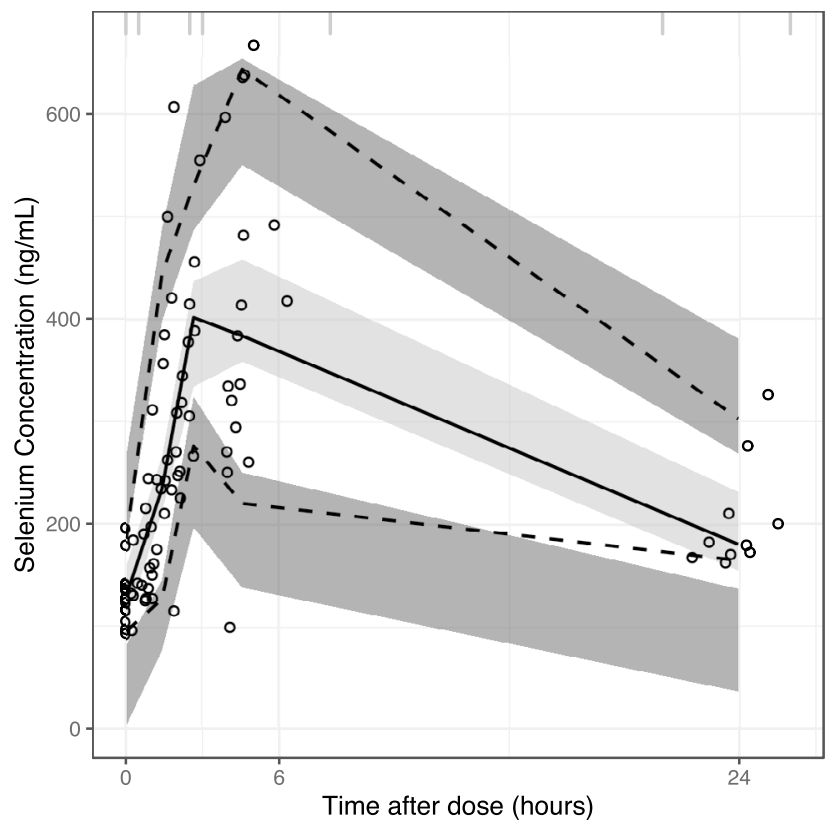

Fig. 3 Visual predictive check of pharmacokinetic model. Simulation-based diagnostic comparing observations (raw data, open circles) and predictions from the pharmacokinetic model. Percentiles for raw data: 97.5 (upper, dashed), 50 (median, solid), 2.5 (lower, dashed). Confidence interval for percentiles of simulated data: 97.5 (upper, dark gray shaded area), 50 (median, light gray shaded area), 2.5 (lower, dark gray shaded area)

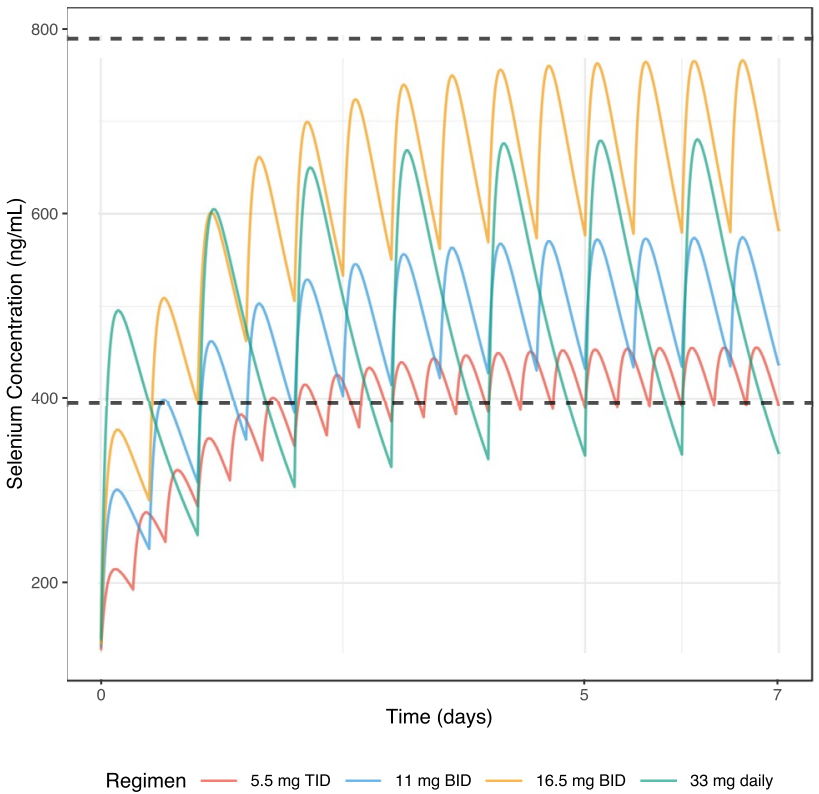

Fig. 4 Proposed oral dosing regimens. Simulated concentration-time profiles using the pharmacokinetic model for selected oral dosing regimens that achieved the target selenium concentration range demarcated by horizontal gray dashed lines (lower, $395 \mathrm{ng} / \mathrm{mL}$; upper, $790 \mathrm{ng} / \mathrm{mL}$ ). BID twice daily, TID three times daily

Table 2 Therapeutic windows: comparison of trough and peak plasma concentrations $(\mathrm{ng} / \mathrm{mL})$ for each proposed dosing regimen of sodium selenite

\begin{tabular}{lll}
\hline Proposed regimen & $C_{\text {trough }}$ & $C_{\text {peak }}$ \\
\hline $5.5 \mathrm{mg}$ TID & 391 & 455 \\
$11 \mathrm{mg}$ BID & 434 & 574 \\
$16.5 \mathrm{mg}$ BID & 579 & 766 \\
$33 \mathrm{mg}$ daily & 339 & 680 \\
\hline
\end{tabular}

$B I D$ twice daily, $C_{\text {peak }}$ peak plasma concentration, $C_{\text {trough }}$ trough plasma concentration, TID three times daily

to characterize selenite pharmacokinetics, which employed several assumptions (e.g., no drug distribution) and had limited predictive power. Our NLMEM approach overcame these limitations to characterize the pharmacokinetic data across five dosing cohorts and with consideration for variability in the patient population. The pharmacokinetic model explored both endogenous and exogeneous contributions to the measured selenium level and presented a mathematical relationship between dose and bioavailability that are absent in prior work. 
Fig. 5 Optimal study design. A Optimal experimental design pharmacokinetic sampling times for three regimens (16.5 mg BID, $11 \mathrm{mg}$ BID, $33 \mathrm{mg}$ daily); model typical values (lines), model typical prediction intervals (shaded area), optimal pharmacokinetic sampling times (filled circles). B Relative standard error of parameter estimates from the original design (gray) and optimized design (dark blue). BID twice daily, PopED Population Experimental Design (version 0.4.0, $\mathrm{R}$ package)

\section{(a)}

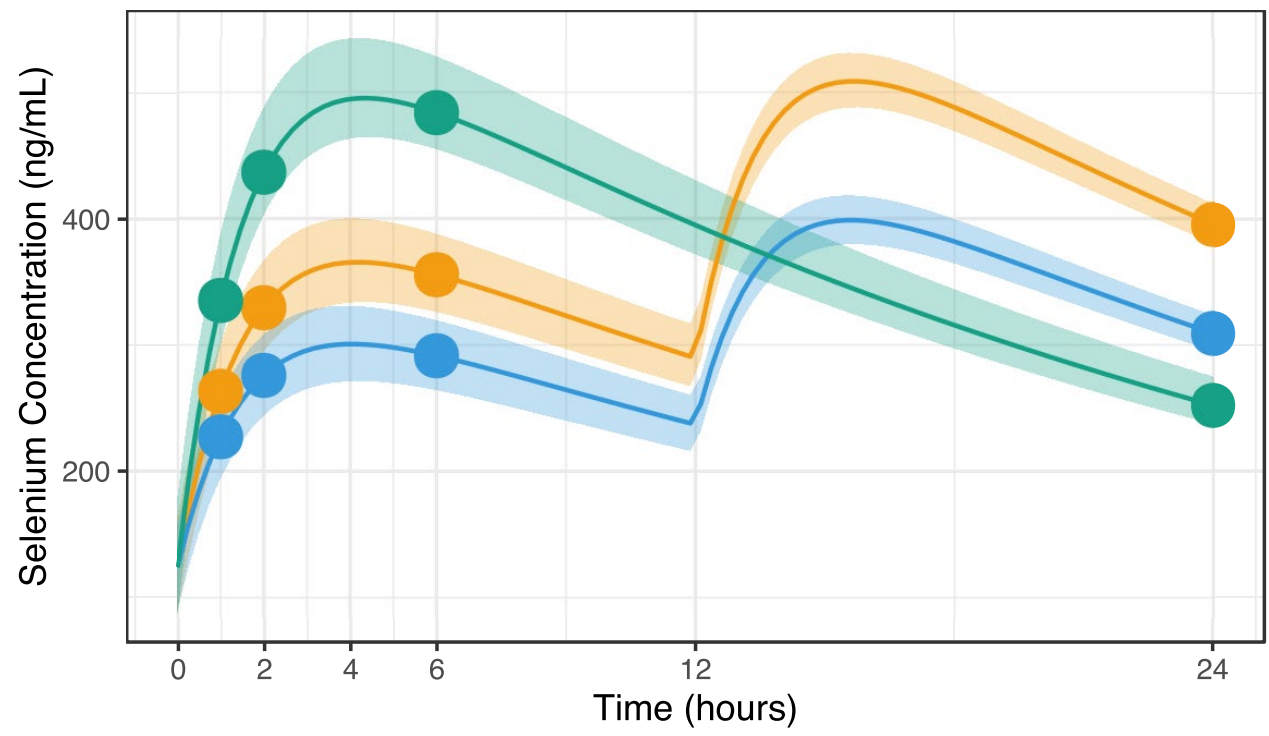

Dosing Cohort

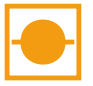
$16.5 \mathrm{mg} \mathrm{BID}$ $11 \mathrm{mg} \mathrm{BID}$ $33 \mathrm{mg}$ daily

(b)

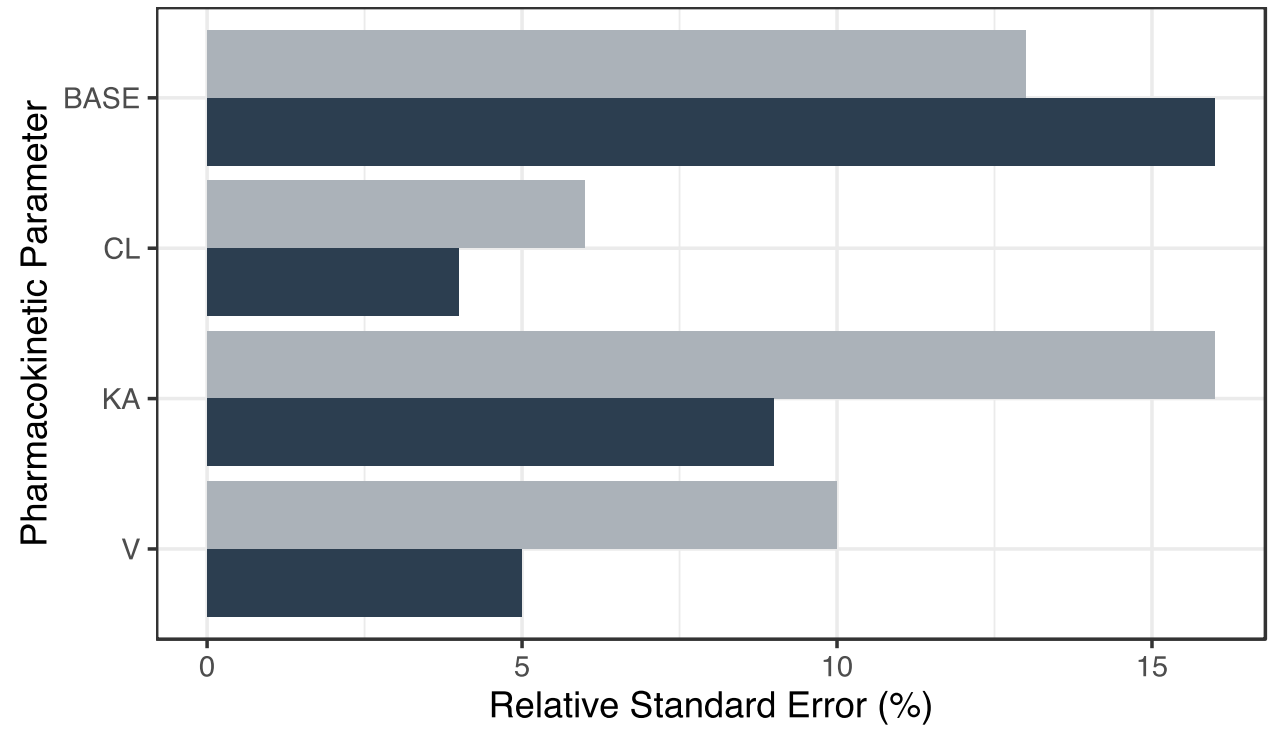

Design

Optimized (from PopED)

Original (from NONMEM)
The trace element selenium, in its organic form, is an important component of antioxidant enzymes and is found in a variety of tissues (e.g., skeletal muscle) when consumed in the diet [5]. Therefore, each individual has a baseline in vivo selenium level. In this clinical study, exogenous doses of selenite contributed to an ex vivo selenium level. In an effort to distinguish in vivo from ex vivo contributions, the baseline selenium level was estimated using the pharmacokinetic model and the pre-dose levels on day 1 in the intensive phase of pharmacokinetic sampling. In the sparse sampling phase of the study, levels returned close to baseline between doses; however, it was not possible to clearly distinguish whether the measured levels were indeed slightly elevated above the baseline level, which is inherently dynamic in a given individual. Therefore, only the intensive pharmacokinetic data were modeled. This decision is appropriate given the sparse 
design beyond the first $24 \mathrm{~h}$ of the study and in the absence of any characterization of the in vivo circadian time course of selenium. These shortcomings are also evidenced in the VPC (Fig. 3), where a model misspecification can be seen in the lower 95\% simulation-based confidence interval in the elimination phase of the pharmacokinetic profile. More time points collected through the elimination phase following exogenous dosing will help better characterize the halflife of selenium and the trough concentrations. Coupled with more information about in vivo selenium dynamics (e.g., circadian time course) in the absence of exogenous dosing, we can also better differentiate the in vivo from ex vivo contributions.

An objective of the modeling exercise was to propose dosing regimens that achieve target therapeutic concentrations. The VPC (Fig. 3) shows that the maximum concentration is described well, allowing the model to serve its intended purpose. The dose-bioavailability expression used to describe the saturable absorption is largely empirical, which is a limitation of the model structure. More pharmacokinetic data from ongoing clinical studies for the dose range investigated herein are needed to better understand the mechanism underlying the observed relationship and to refine the mathematical expression. For drugs exhibiting an inverse relationship, dosing more frequently reduces large fluctuations between peak and trough levels. This concept is illustrated well in the dosing simulations shown in Fig. 1. While daily dosing with 49.5 and $33 \mathrm{mg}$ reached the target in vitro concentration range, significant fluctuations in the peaks and troughs can be seen (Fig. 1b in the ESM). In contrast, the $11 \mathrm{mg}$ BID, $16.5 \mathrm{mg}$ BID, and $5.5 \mathrm{mg}$ TID regimens provided tighter pharmacokinetic profiles (Fig. 1c, $\mathrm{d}$ in the ESM). The in vitro target concentration range will likely be replaced when forthcoming studies provide more relevant pharmacokinetic/pharmacodynamic (PK/PD) indices such as in vivo exposure levels (area under the concentration-time curve) that correspond to clinical efficacy. Upon doing so, these dosing regimen recommendations may be revised and more complex PK/PD relationships may be explored using NLMEM.

Individuals treated with the $33 \mathrm{mg}$ dose level in the phase I study experienced significant (mostly grade 1, some grade 2) gastrointestinal side effects that affected their quality of life [12]. The side effects required medication for symptom control but were manageable. Administering selenite doses with food may readily alleviate some gastrointestinal toxicity and allow higher doses with less discomfort. This study did not evaluate the impact of food on selenite pharmacokinetics; rather, oral doses were administered $2 \mathrm{~h}$ before or after meals. Drug-food interactions may be expected based on what has been observed for other minerals such as iron. For example, iron absorption is significantly impacted by mealtimes, caloric content, and acid content [21]. Therefore, the effect that food may have on oral selenite absorption remains to be investigated and may influence future study design. Given these considerations, of the four proposed regimens, we recommend either $11 \mathrm{mg}$ BID or $16.5 \mathrm{mg}$ BID; both regimens are comparable to one another. Alternatively, individuals may begin with $33 \mathrm{mg}$ daily to quickly reach the lower limit of the target concentration range (395 ng/ $\mathrm{mL}$ ) and then de-escalate to $16.5 \mathrm{mg}$ BID or $11 \mathrm{mg}$ BID if significant gastrointestinal side effects occur. The proposed regimens were restricted to multiples of the available tablet strength $(5.5 \mathrm{mg})$ and to the dose levels tested in the study for which safety profiles are known.

To design a subsequent selenite pharmacokinetic study, clinical trial simulations or experimental design optimization using D-optimality are two approaches that can be applied. The former explores a fixed number of potential designs with large datasets simulated and fitted to each design, which is computationally intensive [20,22]. Experimental design optimization using FIM for population pharmacokinetic models yields informative pharmacokinetic study designs with precise parameter estimates by using maximum likelihood estimation methods to obtain the design variables (parameter estimates) with the highest FIM without the need for intensive simulations $[18,20]$. A number of studies have used PopED to design pharmacokinetic and PK/PD studies in adult and pediatric populations [23-25].

Several design factors contribute to experimental design optimization: number of study subjects, open clinic hours, sampling times, and number of observations per subject. The sampling times for all dose levels and administration frequencies were optimized simultaneously to yield protocolfriendly times that minimized both scheduling challenges for clinic staff and patient inconvenience. Sampling times were limited to within clinic hours and specified in the design space using a range of potential times within the first $12 \mathrm{~h}$ and when the clinic re-opened the next day. The sample size was taken from Bayesian hierarchical modeling and simulations of toxicity scenarios completed for a subsequent clinical trial protocol; a sample size of 25 provided the highest probability of choosing the correct maximum tolerated dose. The number of observations per individual was fixed to four to minimize the number of blood draws. These factors, and many more, are customizable in PopED.

The optimal design exercise evaluated three proposed dosing regimens together to determine the optimal sampling times for a subject who may be placed on any of the regimens during the course of a study. The optimized sampling times are similar to those in the original design, confirming that those sampling times, which were selected with minimal prior knowledge about the pharmacokinetic profile of orally administered selenite, were well chosen. The precision is expected to improve, which it does for the key parameter estimates, but not 
drastically, because the original and optimized sampling times are similar to each other. The precision for the base parameter decreases, but since the original study was not designed to estimate this parameter well, lower precision is expected.

The experimental design optimization approach has some limitations. Most importantly, it relies on prior information, namely a population pharmacokinetic model, parameter estimates, and variability measures, for a clinical study that may not have been designed with the intention of being used for a design optimization exercise [19]. For example, the clinical study data described by the final pharmacokinetic model do not capture the dynamics between ex vivo and in vivo selenium levels. The small study sample size with few time points collected in the elimination phase resulted in a final pharmacokinetic model with no significant estimate of IIV on the key pharmacokinetic parameters. With more pharmacokinetic data from multiple clinical studies, the pharmacokinetic characterization may be strengthened and the variability well described with a covariate analysis, leading to a more robust design.

\section{Conclusions}

In this study, we characterized the clinical pharmacokinetics of orally administered sodium selenite using NLMEM. This modeling approach has improved our understanding of the time course of selenium concentrations in patients with metastatic cancer. With simulation-based methods, we proposed dosing regimens and made recommendations for the optimal sampling times of subsequent studies exploring the pharmacokinetics of selenite as monotherapy or in combination therapy. This work provides the foundation for subsequent model-informed analyses to evaluate the drug concentration-response of selenite in the treatment of metastatic cancers.

Supplementary Information The online version contains supplementary material available at https://doi.org/10.1007/s40268-021-00340-9.

Acknowledgements The authors thank Navjot Sandhu and Stacy Leanne Stamps-DeAnda for their assistance with compiling the pharmacokinetic data from the study.

\section{Declarations}

Funding The clinical trial was supported by the National Institutes of Health National Center for Advancing Translational Sciences (grant UL1 TR001085) and the SPARK Program, Cancer Institute, and Office of Technology and Licensing at Stanford University.

Conflict of interest PJ, SJK, MGC, and RMS have no conflicts of interest that are directly relevant to the content of this article. PJ is sup- ported by grant T32 GM007546 from the National Institute of General Medical Sciences.

Ethical approval All procedures performed in studies involving human participants were in accordance with the ethical standards of the institutional and/or national research committee and with the 1964 Helsinki declaration and its later amendments or comparable ethical standards. The study was approved by the US FDA (IND122151), the Stanford University Internal Review Board, and the Scientific Review Committee for the Stanford Cancer Institute.

Informed consent Informed consent was obtained from all individual participants in the study (NCT02184533).

Authors' contributions PJ, SJK, MGC, and RMS designed the research, performed the research, analyzed the data and wrote the manuscript.

Open Access This article is licensed under a Creative Commons Attribution-NonCommercial 4.0 International License, which permits any non-commercial use, sharing, adaptation, distribution and reproduction in any medium or format, as long as you give appropriate credit to the original author(s) and the source, provide a link to the Creative Commons licence, and indicate if changes were made. The images or other third party material in this article are included in the article's Creative Commons licence, unless indicated otherwise in a credit line to the material. If material is not included in the article's Creative Commons licence and your intended use is not permitted by statutory regulation or exceeds the permitted use, you will need to obtain permission directly from the copyright holder. To view a copy of this licence, visit http://creativecommons.org/licenses/by-nc/4.0/.

\section{References}

1. Balk SP. Androgen receptor as a target in androgen- independent prostate cancer. Urology. 2002;60(3):132-8. https://doi.org/10. 1016/S0090-4295(02)01593-5.

2. NCI Dictionary of Cancer Terms [database on the Internet] 2019. https://www.cancer.gov/publications/dictionaries/cancer-terms/ def/androgen-ablation. Accessed:

3. Nuhn P, De Bono JS, Fizazi K, Freedland SJ, Grilli M, Kantoff $\mathrm{PW}$, et al. Update on systemic prostate cancer therapies: management of metastatic castration-resistant prostate cancer in the era of precision oncology. Eur Urol. 2019;75(1):88-99. https://doi. org/10.1016/j.eururo.2018.03.028.

4. Crawford ED, Higano CS, Shore ND, Hussain M, Petrylak DP. Treating patients with metastatic castration resistant prostate cancer: a comprehensive review of available therapies. J Urol. 2015;194(6):1537-47. https://doi.org/10.1016/j.juro.2015.06.106.

5. Supplements OoD. Selenium. National Institutes of Health 2018. https://ods.od.nih.gov/factsheets/Selenium-HealthProfessional/.

6. Husbeck B, Peehl DM, Knox SJ. Redox modulation of human prostate carcinoma cells by selenite increases radiation-induced cell killing. Free Radic Biol Med. 2005;38(1):50-7. https://doi. org/10.1016/j.freeradbiomed.2004.09.022.

7. Husbeck B, Nonn L, Peehl DM, Knox SJ. Tumor-selective killing by selenite in patient-matched pairs of normal and malignant prostate cells. Prostate. 2006;66(2):218-25. https://doi.org/10.1002/ pros.20337.

8. Husbeck B, Bhattacharyya RS, Feldman D, Knox SJ. Inhibition of androgen receptor signaling by selenite and methylseleninic acid in prostate cancer cells: two distinct mechanisms of action. Mol Cancer Ther. 2006;5(8):2078-85. https://doi.org/10.1158/15357163.MCT-06-0056. 
9. Bhattacharyya RS, Husbeck B, Feldman D, Knox SJ. Selenite treatment inhibits LAPC-4 tumor growth and prostate-specific antigen secretion in a xenograft model of human prostate cancer. Int J Radiat Oncol Biol Phys. 2008;72(3):935-40. https://doi.org/ 10.1016/j.ijrobp.2008.07.005.

10. Corcoran NM, Najdovska M, Costello AJ. Inorganic selenium retards progression of experimental hormone refractory prostate cancer. J Urol. 2004;171(2 Pt 1):907-10. https://doi.org/10.1097/ 01.ju.0000092859.16817.8e.

11. Brodin O, Eksborg S, Wallenberg M, Asker-Hagelberg C, Larsen $\mathrm{EH}$, Mohlkert D, et al. Pharmacokinetics and toxicity of sodium selenite in the treatment of patients with carcinoma in a phase I clinical trial: the SECAR study. Nutrients. 2015;7(6):4978-94. https://doi.org/10.3390/nu7064978.

12. Knox SJ, Jayachandran P, Keeling CA, Stevens KJ, Sandhu $\mathrm{N}$, Stamps-DeAnda SL, et al. Results from a phase 1 study of sodium selenite in combination with palliative radiation therapy in patients with metastatic cancer. Transl Oncol. 2019;12(11):152531. https://doi.org/10.1016/j.tranon.2019.08.006.

13. Al-Taie OH, Seufert J, Karvar S, Adolph C, Mork H, Scheurlen $\mathrm{M}$, et al. Selenium supplementation enhances low selenium levels and stimulates glutathione peroxidase activity in peripheral blood and distal colon mucosa in past and present carriers of colon adenomas. Nutr Cancer. 2003;46(2):125-30. https://doi.org/10. 1207/S15327914NC4602_04.

14. Bruns F, Buntzel J, Mucke R, Schonekaes K, Kisters K, Micke O. Selenium in the treatment of head and neck lymphedema. Med Princ Pract. 2004;13(4):185-90. https://doi.org/10.1159/00007 8313.

15. Buntzel J, Micke O, Kisters K, Bruns F, Glatzel M, Schonekaes $\mathrm{K}$, et al. Selenium substitution during radiotherapy of solid tumours - laboratory data from two observation studies in gynaecological and head and neck cancer patients. Anticancer Res. 2010;30(5):1783-6.

16. Zimmermann T, Leonhardt H, Kersting S, Albrecht S, Range U, Eckelt U. Reduction of postoperative lymphedema after oral tumor surgery with sodium selenite. Biol Trace Elem Res. 2005;106(3):193-203. https://doi.org/10.1385/BTER:106:3:193.

17. Keizer RJ, Karlsson MO, Hooker A. Modeling and simulation workbench for NONMEM: tutorial on Pirana, PsN, and Xpose.
CPT Pharmacometrics Syst Pharmacol. 2013;2:e50. https://doi. org/10.1038/psp.2013.24.

18. Nyberg J, Ueckert S, Stromberg EA, Hennig S, Karlsson MO, Hooker AC. PopED: an extended, parallelized, nonlinear mixed effects models optimal design tool. Comput Methods Progr Biomed. 2012;108(2):789-805. https://doi.org/10.1016/j.cmpb. 2012.05.005.

19. Foracchia M, Hooker A, Vicini P, Ruggeri A. POPED, a software for optimal experiment design in population kinetics. Comput Methods Progr Biomed. 2004;74(1):29-46. https://doi.org/10. 1016/S0169-2607(03)00073-7.

20. Nyberg J, Bazzoli C, Ogungbenro K, Aliev A, Leonov S, Duffull $\mathrm{S}$, et al. Methods and software tools for design evaluation in population pharmacokinetics-pharmacodynamics studies. $\mathrm{Br}$ J Clin Pharmacol. 2015;79(1):6-17. https://doi.org/10.1111/bcp. 12352.

21. Galanello R, Piga A, Cappellini MD, Forni GL, Zappu A, Origa R, et al. Effect of food, type of food, and time of food intake on deferasirox bioavailability: recommendations for an optimal deferasirox administration regimen. J Clin Pharmacol. 2008;48(4):42835. https://doi.org/10.1177/0091270007313327.

22. Holford N, Ma SC, Ploeger BA. Clinical trial simulation: a review. Clin Pharmacol Ther. 2010;88(2):166-82. https://doi.org/10.1038/ clpt.2010.114.

23. Mentre F, Chenel M, Comets E, Grevel J, Hooker A, Karlsson MO, et al. Current use and developments needed for optimal design in pharmacometrics: a study performed among DDMoRe's European federation of pharmaceutical industries and associations members. CPT Pharmacometr Syst Pharmacol. 2013;2:e46. https://doi.org/10.1038/psp.2013.19.

24. Roberts JK, Stockmann C, Balch A, Yu T, Ward RM, Spigarelli $\mathrm{MG}$, et al. Optimal design in pediatric pharmacokinetic and pharmacodynamic clinical studies. Paediatr Anaesth. 2015;25(3):22230. https://doi.org/10.1111/pan.12575.

25. Solans BP. Application and development of computational approaches to optimize treatment of malignancies using routine clinical data. Pamplona: Universidad De Navarra; 2019. 